\title{
RobEcolo: OPTIMAL DESIGN OF A WOODEN FIVE-BAR MECHANISM
}

\author{
Lila Kaci ${ }^{1,2}$, Sébastien Briot ${ }^{1,3}$, Clément Boudaud ${ }^{4}$ and Philippe Martinet ${ }^{2,5}$ \\ ${ }^{1}$ Laboratoire des Sciences du Numérique de Nantes (LS2N), UMR CNRS 6004, Nantes, France \\ 2 École Centrale de Nantes, France \\ ${ }^{3}$ Centre National de la Recherche Scientifique (CNRS) \\ ${ }^{4}$ LIMBHA, Groupe École Supérieure du Bois, Nantes, France \\ ${ }^{5}$ Centre de Recherche INRIA Sophia Antipolis \\ Email: \{Lila.Kaci,Sebastien.Briot,Philippe.Martinet\}@ls2n.fr \\ Email: clement.boudaud@ecoledubois.fr
}

June 26, 2019

\begin{abstract}
Eco-design of robots has almost never be en explored in the past. This work investigates the potential of using bio-sourced materials, which have almost no environmental impact, instead of metals for robot design. Also, wood is one of the best candidates because of its interesting mechanical properties. However, wood performance / dimensions vary with the atmospheric conditions / external solicitations. Thus, it is challenging to design a stiff and accurate wooden industrial robot.

Therefore, the objective of this paper is to describe a new design methodology leading to the design a wooden five-bar mechanism reliable in terms of accuracy and stiffness. The design optimization problem is solved in cascade. The first optimization process proposes to use a control-based design approach in order to compute the optimal primary geometric parameters of the robot (lengths of the links). This approach takes into account the sensor-based controller performance during the design phase. The second optimization process deals with the issue of the variability of the wood mechanical performance. It is
\end{abstract}

based on a reliable topology optimization approach and allows for finding the shape of the robot links for which the impact of this variability in terms of deformation is minimized. Theoretical developments are described, solved and the obtained results allowed the prototyping of an industrial wooden five-bar mechanism.

\section{INTRODUCTION}

Robots considerably impact the environment, not only during their use but also during their manufacturing phase which represents almost $50 \%$ of the impact [1]. The impact of the robot manufacturing is due to the use of polluting materials such as, for the design of the mechanical architecture, metals (iron or aluminum alloys) or even carbon composites. However, as shown during COP21, one of the priority in the world is the Climate Change Mitigation. Thus, it is very urgent to find new solutions to design machines that limit the Environmental Impact (EI) of the human industrial activities. Robotics must also participate to this effort and robot designers should 
investigate the field of robot eco-design.

In order to decrease the EI during the design of the robot architecture, bio-sourced materials (BSM) could be used. BSM have a little (even no) EI $[2,3]$. In the past, wood was used in machine design, (e.g. planes up to Wold War II, cars and aircraft chassis [4]). Nowadays, wood is still popular in building design [5] due to its high mechanical stiffness and its low cost. In Robotics, the use of this material is limited to design mock-ups and prototypes [6,7]. A first attempt to use wood in robot design was presented in [3]. The obtained results validated the feasibility of designing industrial wooden robot. However, the wood was not used to guarantee the robot performance in terms of stiffness and accuracy. This is a crucial issue because wood is an organic material, and it presents a variability in its performance / dimensions with respect to atmospheric conditions / external solicitations. Its performance also depends on the conditions in which trees have grown $[8,9]$.

In this paper we introduce a design methodology well adapted in order to design a stiff and accurate wooden industrial robot. This methodology is applied on a five-bar mechanism, i.e. a parallel robot with two translational degrees of freedom. This methodology is based on the following concepts:

- In order to ensure the robot accuracy even with the variability in the wooden link dimensions, sensor-based controllers could be used [10]. However, as shown in [11], positioning accuracy of sensor-based controllers (obviously) depends of the number and types of sensors that are used for the robot control, but also, in certain cases, of the robot geometric parameters [12]. Therefore, the robot design process must take into account performance criteria coming from usual physical properties [13] but also from the type of controller that will be implemented on the robot. This approach is quite unusual for industrial robot design.

- In order to ensure the robot stiffness performance even with the variability of the wood mechanical properties, link shapes can be optimized thanks to reliable topology optimization algorithms [14].
This methodology has been defined in the context of the project RobEcolo [15] whose goal is to show that the EI of robots can be considerably reduced by using BSM instead of metals or carbon composites.

This paper is divided as follows. In Section 2, the robot design requirements are specified. Section 3 introduces the sensor-based control approach that will be used to control the robot motions and presents the design methodology adapted to optimize the primary geometric parameters of the wooden five-bar mechanism taking into account the controller characteristics. In Section 4, a reliable topology optimization approach able to find the robot link shapes is proposed. Section 5 will describe the CAD design and manufacture of the prototype of the wooden fivebar mechanism. Finally, in section 6 , conclusions are drawn.

\section{ROBOT ARCHITECTURE AND SPECIFICATIONS}

The five-bar mechanism geometry [16] is illustrated in Fig. 1. It is a two degrees-of-freedom (dof) planar parallel robot with two translations in the plane $\left(\mathbf{x}_{0} O \mathbf{y}_{0}\right)$. The end-effector is positioned at point $C$. The mechanism consists of four links: proximal links are the bodies between $A_{i}$ and $B_{i}(i=1,2)$ while the bodies between points $B_{i}$ and $C$ are called distal links. The proximal and distal links are connected by three passive revolute joints, the joints located at point $A_{1}$ and $A_{2}$ are active.

The requirements that the wooden five-bar mechanism should satisfy have been fixed by our partners in the project RobEcolo [15]. These requirements are given in Tab. 1. These performance should be valid wherever in the robot workspace. Thus, of course, the workspace should be free of singularity. In addition, the robot must be as compact as possible.

Deformations along $\mathbf{z}_{0}$ have not been specified because the robot purpose is to have a precise positioning in its plane of motion. However, these vertical deformations will be checked a posteriori in the design process in order to verify that they are "acceptable", which means for the project partners, around 


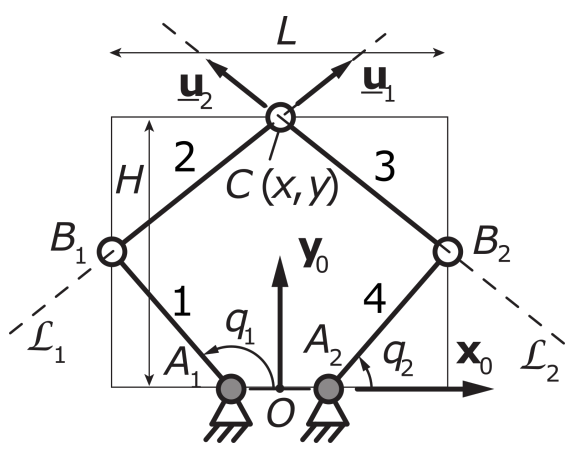

Figure 1: Five-bar mechanism

Table 1: Specifications for the Wooden Five-bar Mechanism

\begin{tabular}{ll}
\hline \hline $\begin{array}{l}\text { Absolute positioning accu- } \\
\text { racy }\end{array}$ & $0.5 \mathrm{~mm}$ \\
Regular workspace size & $800 \mathrm{~mm} \times$ \\
& $200 \mathrm{~mm}$ \\
End-effector deformations in & $0.15 \mathrm{~mm}$ in \\
the plane $\left(\mathbf{x}_{0} O \mathbf{y}_{0}\right)$ under & translation in the \\
loadings $\mathbf{f}_{1}=\left[F_{x}=\right.$ & plane $\left.\left(\mathbf{x}_{0} O \mathbf{y}_{0}\right)\right)$ \\
$150 N F_{y}=0 N M_{z}=$ & and $0.25 \mathrm{mrad}$ in \\
$-1 N m]^{T}$ and $\mathbf{f}_{2}=\left[F_{x}=\right.$ & rotation around \\
$0 N F_{y}=150 N M_{z}=$ & $\mathbf{z}_{0}$ \\
$1 \mathrm{Nm}]^{T}$ & \\
\hline \hline
\end{tabular}

500 microns under a loading of $10 \mathrm{~N}$ along $\mathbf{z}_{0}$.

The robot is a prototype made for validating performance in terms of stiffness and accuracy, therefore no dynamic properties have been imposed. However, the design process should tend to reduce the mass or robot link inertia so that it avoids leading to the manufacture of bulky links.

Finally, the number and types of sensor has been imposed by the project partners. With respect to the desired 500 microns of absolute positioning accuracy specified in Tab. 1, we propose to use four cameras Toshiba Bu 238 M $(1920 \times 1200$ pixels of resolution and a focal length of $8 \mathrm{~mm}$ ) as sensors. With lens of $8 \mathrm{~mm}$, distortion will be limited and the global visual system (4 cameras) will require to be calibrated for the real implementation. Then, to control the robot, servoing approaches will be used. These approaches are relevant with respect to the accuracy to be reached [10].

Based on all these requirements, it is possible to see that the problem can be solved in cascade. Indeed, the absolute positioning accuracy requirements essentially depend on the robot link length and camera performance / position, while the deformations is more related to the shape of the robot links and material properties. Therefore, the optimization problem will be separated into two independent parts: the first one will deal with the definition of the primary geometric parameters, i.e. the link lengths and camera positions/orientations, under robot positioning error specifications; the second one will deal with the definition of the optimal link shapes under elastic performance constraints.

The next Section introduces the first optimization problem taking into account the characteristics of the controller and of the perception system.

\section{CONTROL-BASED DE- SIGN}

\subsection{Brief recalls on leg-based visual servoing}

As mentioned above, in order to ensure the robot positioning accuracy even with the dimensional variability of the wooden links, visual servoing will be used. The usual approaches are based on the direct observation of the end-effector pose [17]. In our case however the end-effector of a five-bar mechanism is a point, which is difficult to observe. This is why we decided to observe the distal links, whose dimensions are more compatible with an observation by a camera, as it was proposed in $[18,19]$. The scheme of a general vision-based control based on the observation of the robot links is presented in Fig. 2. However, it is known from [12] that these types of controllers present singularities, that affect their accuracy performance. These singularities depend not only on the choice of the sensor localizations but also on the robot dimensions. Thus, it is highly necessary to take into account all these parameters in the design process.

In order to facilitate the observation of the distal links, in the following of the paper, their shapes 


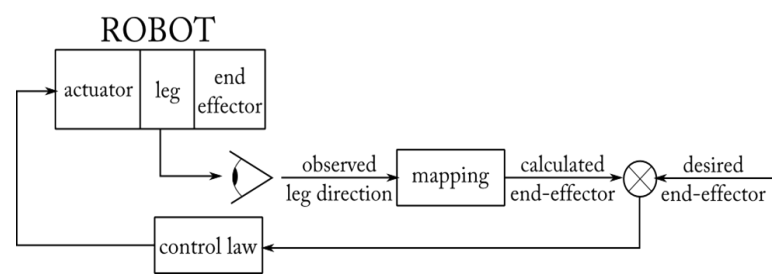

Figure 2: A general sensor-based control scheme

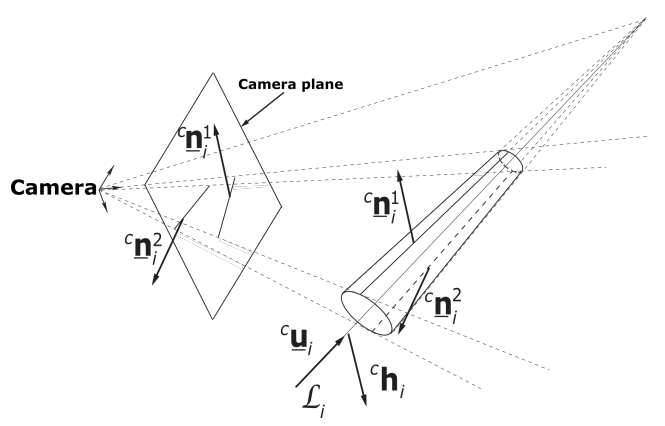

Figure 3: Projection of a cylinder in the camera plane

are imposed to be cylindrical [18]. Indeed, as shown in Fig. 3, cylinder projections in the camera plane are two lines whose interpretation planes (i.e. the planes passing through the camera center and the lines) have as normal vectors ${ }^{c} \dot{\underline{\mathbf{n}}}_{i}^{1}$ and ${ }^{c} \underline{\dot{\mathbf{n}}}_{i}^{2}$ (the superscript ${ }^{c}$ denotes that these vectors are expressed in the camera frame). Their expressions depend on the cylinder size and location. These two vectors are indeed the measurements that can be extracted from the camera observation. By knowing them and using the expressions given in [18], it is then possible to reconstruct the Plücker coordinates $\left({ }^{c} \underline{\mathbf{u}}_{i},{ }^{c} \mathbf{h}_{i}\right)$ of the line $\mathcal{L}_{i}$ passing trough the cylinder axis and their derivatives with respect to time as:

$$
\left[\begin{array}{ll}
{ }^{c} \underline{\dot{\mathbf{u}}}_{i}^{T} & { }^{c} \dot{\mathbf{h}}_{i}^{T}
\end{array}\right]^{T}=\mathbf{H}_{i}^{T}\left[\begin{array}{l}
{ }^{c} \underline{\dot{\mathbf{n}}}_{i}^{1} \\
{ }^{c} \underline{\dot{\mathbf{n}}}_{i}^{2}
\end{array}\right], \quad i=1,2
$$

where $\mathbf{H}_{i}^{T}$ is a $(6 \times 6)$ matrix whose rank is equal to 4 [18]. In this expression, the derivatives $\left({ }^{c} \underline{\dot{\mathbf{u}}}_{i},{ }^{c} \dot{\mathbf{h}}_{i}\right)$ of the Plücker coordinates represent the velocity of displacement of the observed distal link $i$. Moreover, as shown in $[18,20]$ the relationship between the fivebar end-effector twist ${ }^{c} \boldsymbol{\tau}_{c}$ and the velocities of the distal link $i(i=1,2)$ is given by:

$$
\left[\begin{array}{ll}
{ }^{c} \underline{\dot{\mathbf{u}}}_{i}^{T} & { }^{c} \dot{\mathbf{h}}_{i}^{T}
\end{array}\right]^{T}=\mathbf{M}_{i}^{T c} \boldsymbol{\tau}_{c}
$$

where $\mathbf{M}_{i}^{T}$ is the interaction matrix relating the velocity of the distal link $i$ to the velocity of the endeffector. This interaction matrix is of dimension $(6 \times 6)$ with a rank $r \leq 4[18,20]$. From equations (1) and (2), the interaction matrix define the relationship between ${ }^{c} \boldsymbol{\tau}_{c}$ and the vectors ${ }^{c} \underline{\dot{\underline{n}}}_{i}^{j}$ can be found:

$$
\mathbf{H}^{T c} \underline{\dot{\mathbf{n}}}_{k}=\mathbf{M}^{T c} \boldsymbol{\tau}_{c}
$$

with $\mathbf{H}=\left[\begin{array}{ll}\mathbf{H}_{1} & \mathbf{H}_{2}\end{array}\right], \quad \mathbf{M}=\left[\begin{array}{ll}\mathbf{M}_{1} & \mathbf{M}_{2}\end{array}\right], \quad{ }^{c} \underline{\underline{\mathbf{n}}}_{k}=$ $\left[{ }^{c} \underline{\dot{\mathbf{n}}}_{1}^{1 T}{ }^{c} \underline{\dot{\mathbf{n}}}_{2}^{1 T}{ }^{1 T} \dot{\mathbf{n}}_{1}^{2 T}{ }^{2} \dot{\mathbf{n}}_{2}^{2 T}\right]^{T}$. Finally, the using of the pseudo-inverse $\mathbf{M}^{T+}=\left(\mathbf{M} \mathbf{M}^{T}\right)^{-1} \mathbf{M}$ aims at finding the following interaction model for a single camera $k$ :

${ }^{c} \boldsymbol{\tau}_{c}=\mathbf{S}_{k}^{T c} \underline{\dot{\mathbf{n}}}_{k}$, with $\mathbf{S}_{k}^{T}=\mathbf{M}^{T+} \mathbf{H}^{T}=\left(\mathbf{M M}^{T}\right)^{-1} \mathbf{M} \mathbf{H}^{T}$

Then, the interaction model for the system made with four cameras (as in our application) is obtained by:

$$
{ }^{0} \boldsymbol{\tau}_{c}=\mathbf{S}_{t o t}^{T}{ }^{c} \underline{\dot{\mathbf{n}}}_{t o t}
$$

where ${ }^{0} \boldsymbol{\tau}_{c}$ is the end-effector velocity in the world frame, $\mathbf{S}_{\text {tot }}^{T}=\left[{ }^{0} \mathbf{S}_{1},{ }^{0} \mathbf{S}_{2}, \ldots,{ }^{0} \mathbf{S}_{4}\right]^{T}$ in which the matrix ${ }^{0} \mathbf{S}_{k}$ is the interaction matrix associated with the camera $k$ but expressed in the world frame and ${ }^{c} \underline{\mathbf{n}}_{t o t}=\left[{ }^{c} \underline{\dot{\mathbf{n}}}_{1}^{T},{ }^{c} \underline{\mathbf{n}}_{2}^{T}, \ldots, \underline{\mathbf{n}}_{4}^{T}\right]$.

\subsection{Positioning error modelling}

Because a visual servoing will be used in order to ensure the positioning accuracy of the robot, the position error comes from the camera observation error and no more from the error of the encoder measurements (as it is the case in classic encoder-based control schemes [21]). In a real-life scenario, the observation errors appear due to noise in the image. We modeled this noise by a random shift in the pixels where the image projection of the links edges meet the frame boundary (Fig. 4). In our design process, an error of 0.1 pixel was considered: it can indeed be obtained by using subpixellic observation approaches such as in [22] and can straightforwardly be related to a variation $\delta \underline{\mathbf{n}}_{t o t}$ of the measurement vector ${ }^{c} \underline{\dot{n}}_{t o t}$. 


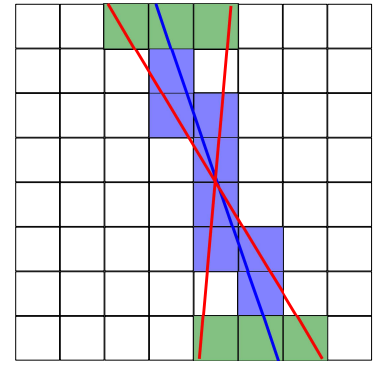

Figure 4: Result of a one-pixel error on the intersection of the image boundary and the observed line

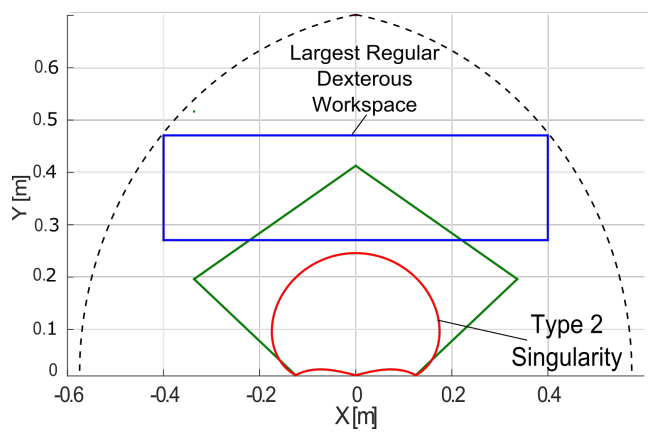

Figure 5: Largest regular dexterous workspace of the optimized five-bar mechanism

The error of observation being small, we approximated the error model relating the variation of the end-effector position $\delta \mathbf{x}$ to the variation $\delta \underline{\mathbf{n}}_{t o t}$ of the measurement vector by using the following first order approximation based on the use of the interaction matrix given at (5):

$$
\delta \mathbf{x}=\mathbf{S}_{t o t}^{T} \delta \underline{\mathbf{n}}_{t o t}
$$

This error model will be used in order to characterize the robot accuracy during the design optimization process.

\subsection{Control-based Design: problem formulation}

After defining the error model, the optimization problem for finding the link lengths and camera locations can be formulated. The objective will be to minimize the size of the five-bar mechanism footprint in the plane (Fig. 1). Moreover, we take into consideration the performance of the controller in term of accuracy. Thus, we formulated the following optimization problem:

$$
\begin{aligned}
\operatorname{minimize} & A=L H \\
\text { over } & \mathbf{x} \\
\text { subject to } & \ell_{W_{x}}>\ell_{W_{x 0}} \text { and } \ell_{W_{y}}>\ell_{W_{y 0}}
\end{aligned}
$$

where: $\mathbf{x}=\left[\begin{array}{lllllll}\ell_{0} & \ell_{1} & \ell_{2} & \mathbf{x}_{c 1} & \mathbf{x}_{c 2} & \mathbf{x}_{c 3} & \mathbf{x}_{c 4}\end{array}\right]$ with $\ell_{0}=$ $\ell_{A_{1} A_{2}}, \ell_{1}=\ell_{A_{1} B_{1}}=\ell_{A_{2} B_{2}}$ and $\ell_{2}=\ell_{B_{1} C}=\ell_{B_{2} C}$ are the robot link lengths, and $\mathbf{x}_{c i}=\left[\begin{array}{lllll}x_{c} & y c & z c & \phi & \theta\end{array}\right]^{T}$ $(i=1$ to 4$)$ is a vector defining the position and orientation of the cameras: $x_{c}, y_{c}$ and $z_{c}$ defining the position of the camera respect to the word frame, $\phi$, $\theta$ and $\psi$ are the ZXZ Euler angles characterizing its orientation. $A=L H$ is the robot footprint (Fig. 1). $\ell_{W_{x}}$ and $\ell_{W_{y}}$ are the dimensions along $\mathbf{x}_{0}$ and $\mathbf{y}_{0}$ of the rectangular dexterous workspace in which all performance must be satisfied [23]. In our case, the robot must have a rectangular dexterous workspace of rectangular shape with dimensions along $\mathbf{x}_{0}$ bigger than $\ell_{W_{x 0}}=800 \mathrm{~mm}$ and along $\mathbf{y}_{0}$ of $\ell_{W_{y 0}}=200 \mathrm{~mm}$ in which the following properties are certified:

1. no Type 2 singularities of the robot and singularities of the controller,

2. the end-effector is within the image frame for all cameras and thus the legs can be observed,

3. knowing the resolution of the cameras, the resolution of the end-effector position should be lower than $0.5 \mathrm{~mm}$. The end effector resolution is computed based on the error model given by (6).

4. the static forces exerted into the passive joints are proportional to $1 / \sin \xi, \xi$ being the angle between the distal links [24]. Consequently, it is decided that $\sin \xi$ should be higher than 0.1 to avoid excessive efforts in the joints.

It is necessary to mention that the error model also depends on the diameter of the cylindrical distal links which was not included in our optimization problem in order to accelerate the computation of the results. This diameter was fixed at $80 \mathrm{~mm}$. 


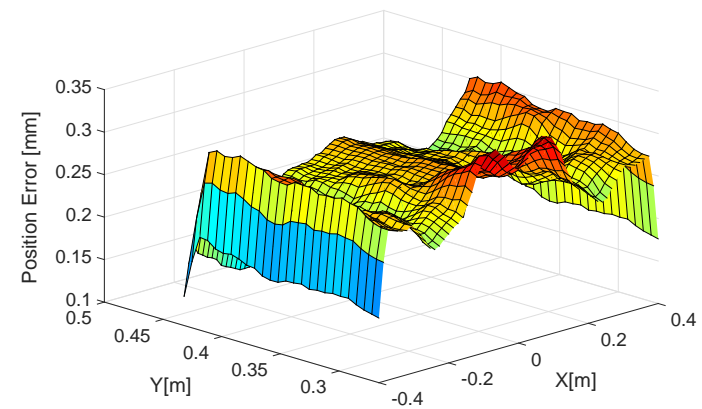

Figure 6: Resolution of the end-effector pose in the LRDW

Table 2: Optimal Design Solution of the Wooden Five-bar Mechanism

\begin{tabular}{l|lll}
\hline \hline$A\left[\mathrm{~m}^{2}\right]$ & $l_{0}[\mathrm{~m}]$ & $l_{1}[\mathrm{~m}]$ & $l_{2}[\mathrm{~m}]$ \\
\hline 0.1372 & 0.125 & 0.280 & 0.400 \\
\hline \hline
\end{tabular}

The optimal design variables and the optimal position / orientation of the cameras can be seen in Tab. 2 and Tab. 3 respectively. It should be mentioned that the position/orientation of cameras 1 and 2 is obtained by symmetry with respect to the $\mathbf{y}_{0}$ axis. Moreover, solving the problem (7) allows to find the position of the largest dexterous regular workspace (Fig. 5). We can clearly observe that it is free of the singularities of the robot (and also of the controller, as they are identical for this type of visual servoing, as shown in [20]). The link lengths given in Tab. 2 are the inputs of the next optimization process.

The value of the positioning end-effector errors in the dexterous regular workspace is illustrated in Fig. 6. These errors are lower that the admissible ones that were fixed at $0.5 \mathrm{~mm}$. Moreover as can be seen in Fig. 6, the error resolution is worsen near the singularities of the robot identical to those of the controller, as mentioned above.
Table 3: Optimal Position and Orientation of Cameras

\begin{tabular}{l|llllll}
\hline \hline Pos/Ori & $x_{c}[\mathrm{~m}]$ & $y_{c}[\mathrm{~m}]$ & $z_{c}[\mathrm{~m}]$ & $\phi[\mathrm{rad}]$ & $\theta[\mathrm{rad}]$ & $\psi[\mathrm{rad}]$ \\
\hline Camera3 & 0.1 & 0.3 & 0.5 & 0 & $\pi$ & 0 \\
\hline Camera4 & 0.1 & 0.5 & 0.5 & 0 & $\pi$ & 0 \\
\hline \hline
\end{tabular}

\section{TOPOLOGY OPTIMIZA- TION}

Topology optimization aims at finding the link shapes for a mechanical structure by optimally distributing the material placement in order to satisfy performance indices: in the literature, a classical problem is to lower the link mass under compliance requirements [25]. In order to compute the link elastic performance, finite element method (FEM) is classically used [26]. Based on this physical model, the extraction of some valuable indices to be included into the optimization process must be made, as well as their gradients with respect to the decision variables [27]. These indices and their gradients are inputs of the optimization solvers among which we can mention the Method of Moving Asymptotes (MMA) [28], the Optimality Criteria (OC) (for instance, see [29]), the Projected Gradient [30], the Convex Linearization method (CONLIN) [31]) and the Linearization Method (LM) [32,33].

In what follows, we first briefly introduce the way the finite element models are made. Then we define a performance index in order to take into account the variability into the wood mechanical behavior. Finally, we formulate the optimization problem and we solve it.

\subsection{Modeling of the linkages elastic behavior}

In what follows, we consider the finite element modelling of the five-bar mechanism, i.e. a robot made of four bodies (Fig. 1). The body $i(i=1, \ldots, 4)$ is meshed with $m_{i}$ elements. The element $j$ of the body $i$ is denoted as the element $i j$ (Fig. 7). To this element, we associate a variable $\rho_{i j}=1$ representing the density of the portion of material linked to 


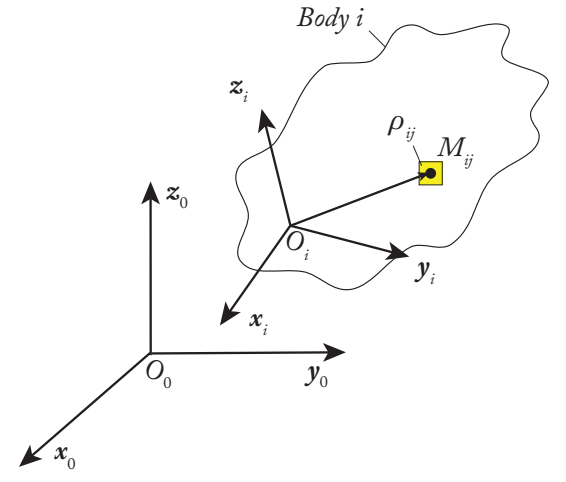

Figure 7: A body in space meshed with finite elements.

the element. These density variables are the decision variables of the optimization problem: densities $\rho_{i j}$ equal to 1 will represent the presence of materials while null densities will represent the absence of materials. A challenge in topology optimization is to avoid having a final link design without too many intermediate values $\left(0<\rho_{i j}<1\right)$ that are difficult to manage by the designer.

In order to solve this problem, FEM is usually combined with an interpolation scheme which is used in order to define an artificial material. This method is called the Solid Isotropic Material with Penalization (SIMP, [34]). This approach has proved to be efficient and it is probably the most widely used material interpolation scheme in topology optimization. This interpolation scheme is adopted in our work.

The SIMP scheme used to parameterize the Young's modulus value $E_{i j}$ of the element $i j$ leads to the following expression:

$$
E_{i j}=E_{\min }+\rho_{i j}^{p}\left(E_{0}-E_{\min }\right), \text { with } \rho_{i j} \in[0,1]
$$

where $p$ is the penalization factor (classically $p=3$ ), $E_{0}$ is the real elasticity modulus of the material and $E_{\min }$ is a very small value. $E_{\min }$ is assigned to regions without materials in order to avoid singularity of the stiffness matrix.

Then, based on this definition of the elasticity modulus for the element $i j$, it is possible to build its stiffness matrix. Once all elementary matrices are de- fined, the computation of the body and robot stiffness matrices is the same as in the traditional methodology [35].

Then, based on these stiffness models, the elastic performance of the mechanism can be defined: in what follows, we are going to consider the deformations $\mathbf{u}_{e}$ of the five-bar mechanism at its end-effector which are linked to the wrench $\mathbf{f}_{e}$ applied on the endeffector by:

$\mathbf{f}_{e}=\mathbf{K}_{e}\left(\boldsymbol{\pi}_{E}, \mathbf{q}\right) \mathbf{u}_{e}\left(\boldsymbol{\pi}_{E}, \mathbf{q}\right) \Rightarrow \mathbf{u}_{e}\left(\boldsymbol{\pi}_{E}, \mathbf{q}\right)=\mathbf{K}_{e}^{-1}\left(\boldsymbol{\pi}_{E}, \mathbf{q}\right) \mathbf{f}_{e}$

in which $\mathbf{K}_{e}\left(\boldsymbol{\pi}_{E}, \mathbf{q}\right)$ is the reduced stiffness matrix characterizing the overall stiffness of the assembled robot (when variable loads are applied at the endeffector location only) $[33,36]$ which depends of:

- the robot configuration $\mathbf{q}$,

- the link Young's moduli (which can be different for any wooden link) which are stacked into the vector $\boldsymbol{\pi}_{E}$. For the five-bar mechanism, this vector has four components.

It should be mentioned that:

- due to page limitation constraints, the assembly of the body and robot stiffness matrices is not defined here, but expressions leading to the full robot model can be found in [33],

- in order to considerably decrease the computational time of the topology optimization algorithm that can be very long for the design of a robot, it is preferable to take advantage of model reduction techniques [36], such as was done in [33].

\subsection{Modelling of the variability in the robot deformations due to the use of wood material}

Choosing a proper wood for the design of our industrial wooden robot is of the utmost importance. It must have a very good stiffness-to-mass ratio while having the lowest dimensional variability due to humidity. This is the reason why we decided to design our links with a novel type of wood named acetylated 
beech [37]. This wood is known to have many advantages over raw wood species in terms of durability and dimensional stability. Moreover, the acytelation is a environmental-friendly chemical treatment [37] which is extremely important with respect to our eco-design purpose.

Wood is an orthotropic material, i.e. it has not the same mechanical behavior depending if we apply loadings along its longitudinal, radial, or tangential direction, the longitudinal direction being along the wood fiber direction. According to the theory of elasticity, the wood is thus parameterized by three Young's moduli $\left(E_{L}, E_{R}\right.$ and $\left.E_{T}\right)$, six Poisson's ratios $\left(\nu_{L R}, \nu_{L T}, \nu_{R T}, \nu_{R L}, \nu_{T L}, \nu_{T R}\right)$ and three shear moduli $\left(G_{L R}, G_{L T}, G_{R T}\right)$. However, the topology optimization is performed on an isotropic model for two reasons:

- the links of parallel robot presents a slenderness corresponding in the longitudinal direction of the wood and these links mostly resist to bending moment, therefor the $E_{L}$ modulus is used. Also, this explain why the value for Poisson's ratio is the mean value $s$ the mean value of the two transversal direction $(R$ and $T) \nu=\frac{\left(\nu_{L R}+\nu_{L L}\right)}{2}$.

- even if the wood is orthotropic, we have the possibility during the manufacture phase to properly design links so that they have a behavior close to the isotropy. This adequate design will be explained in Section 5.

Acetylated beech, as any other type of wood, presents a normal distribution of its longitudinal Young's modulus. Let us define as $\mathbb{E}($.$) the ex-$ pectation operator and $\sigma($.$) the standard deviation$ operator: the expectation of the Young's modulus for the acetylated beech is equal to $\mathbb{E}(E)=\bar{E}=$ $12772 \mathrm{MPa}$ while its standard deviation is equal to $\sigma(E)=2043.5 \mathrm{MPa}[38]$. The Poisson's ratio $\nu$ is considered to be constant and equal to 0.3 [8].

Thanks to this knowledge, it is thus possible to compute the expectation vector $\mathbb{E}\left(\mathbf{u}_{e}\right)$ and the covariance matrix $\operatorname{Cov}\left(\mathbf{u}_{e}\right)$ associated with the endeffector deformations $\mathbf{u}_{e}$, by using the expressions given in [14]:

$$
\begin{aligned}
\operatorname{Cov}\left(\mathbf{u}_{e}\right)= & \sum_{i} \sum_{j} \mathbf{u}_{e i} \mathbf{u}_{e j}^{T} \sigma_{i j} \sum_{i} \sum_{j} \mathbf{u}_{e i j} \sigma_{i j} \\
& +\frac{1}{2} \sum_{i} \sum_{j} \sum_{k}\left(\mathbf{u}_{e i} \mathbf{u}_{e j k}^{T}+\mathbf{u}_{e i j} \mathbf{u}_{e k}^{T}\right) \sigma_{i j k} \\
& +\frac{1}{4} \sum_{i} \sum_{j} \sum_{k} \sum_{l} \mathbf{u}_{e i j} \mathbf{u}_{e k l}^{T}\left(\sigma_{i j k l}-\sigma_{i j} \sigma_{k l}\right)
\end{aligned}
$$

where

- $\mathbf{u}_{e 0}$ is the deformation of the end-effector given by the expression (9) evaluated when the vector of Young's moduli $\boldsymbol{\pi}_{E}$ for the five-bar mechanism is equal to $\pi_{E 0}=[\bar{E} \bar{E} \bar{E} \bar{E}]^{T}$,

- $\mathbf{u}_{e i}=\left.\frac{\partial \mathbf{u}_{e}}{\partial E_{i}}\right|_{\boldsymbol{\pi}_{E 0}}, \mathbf{u}_{e i j}=\left.\frac{\partial^{2} \mathbf{u}_{e}}{\partial E_{i} \partial E_{j}}\right|_{\boldsymbol{\pi}_{E 0}}$, in which $E_{i}$ ( $E_{j}$, resp.) is the Young's modulus of the $i$ th ( $j$ th, resp.) robot link. Detailed expressions of $\mathbf{u}_{e i}$ and $\mathbf{u}_{e i j}$ can be found in [14].

- $\sigma_{i j}=\mathbb{E}\left[\left(E_{i}-\bar{E}\right)\left(E_{j}-\bar{E}\right)\right]$,

- $\sigma_{i j k}=\mathbb{E}\left[\left(E_{i}-\bar{E}\right)\left(E_{j}-\bar{E}\right)\left(E_{k}-\bar{E}\right)\right]$,

- $\sigma_{i j k l}=\mathbb{E}\left[\left(E_{i}-\bar{E}\right)\left(E_{j}-\bar{E}\right)\left(E_{k}-\bar{E}\right)\left(E_{l}-\bar{E}\right)\right]$

It should be mentioned that expressions (10) and (11) are approximated functions obtained thanks to a Taylor series expansion at the order two of the expression of the robot end-effector deformation $\mathbf{u}_{e}$ with respect to the components of the vector $\boldsymbol{\pi}_{E}$ [14]. We tested their validity and our results shown that, for the mechanical parameters of the acetylated beech, the error of approximation is lower than $10 \%$.

Based on these expressions, it is thus possible to define the following constraints representing the possibility of the robot to have reliable stiffness performance:

$$
\begin{aligned}
& \mathbf{u}_{d}\left(\mathbf{f}_{e}, \mathbf{q}, k\right) \leq u_{\max } \\
& \mathbf{u}_{r}\left(\mathbf{f}_{e}, \mathbf{q}, k\right) \leq \theta_{\max }
\end{aligned}
$$

where

$$
\mathbf{u}_{d}\left(\mathbf{f}_{e}, \mathbf{q}, k\right)=\mathbb{E}\left(\left\|\mathbf{u}_{d e}\right\|\right)+k \sigma\left(\left\|\mathbf{u}_{d e}\right\|\right)
$$




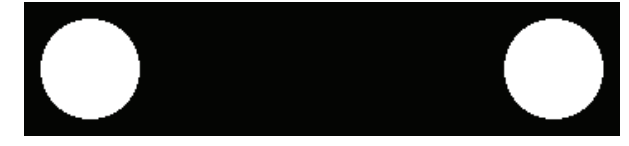

Figure 8: Design of the proximal links: initial design domain

$$
\mathbf{u}_{r}\left(\mathbf{f}_{e}, \mathbf{q}, k\right)=\mathbb{E}\left(\left\|\mathbf{u}_{r e}\right\|\right)+k \sigma\left(\left\|\mathbf{u}_{r e}\right\|\right)
$$

in which

- $\mathbf{u}_{d e}$ are the components of $\mathbf{u}_{e}$ characterizing the end-effector translational deformations due to the loading $\mathbf{f}_{e}$, while $\mathbf{u}_{r e}$ are the components of $\mathbf{u}_{e}$ characterizing the end-effector rotational deformations under the same loading,

- the functions $\sigma\left(\left\|\mathbf{u}_{d e}\right\|\right)$ and $\sigma\left(\left\|\mathbf{u}_{r e}\right\|\right)$ representing the standard deviations of the end-effector translational and rotational deformations, respectively, can be obtained by the following expressions:

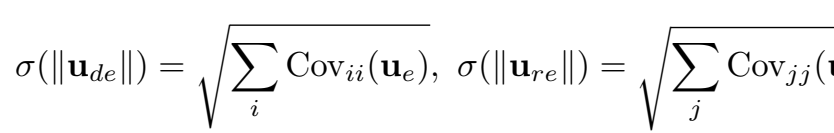

in which $\operatorname{Cov}_{i i}\left(\mathbf{u}_{e}\right)\left(\operatorname{Cov}_{j j}\left(\mathbf{u}_{e}\right)\right.$, resp. $)$ represents all diagonal terms of the covariance matrix $\operatorname{Cov}\left(\mathbf{u}_{e}\right)$ corresponding to the translational (rotational, resp.) components of $\mathbf{u}_{e}$,

- $k$ is a positive real number.

The constraints (12) and (13) are derivated from the well-known Bienaymé-Tchebichev theorem, which states that the probability, for a stochastic variable $X$, to have $|X-\mathbb{E}(X)| \geq k$, is lower than $\frac{\sigma(X)^{2}}{k^{2}}$. Thus, by increasing the value of $k$ in (12) and (13), we reduce the probability to have a robot whose deformations can overpass the requested thresholds $u_{\max }$ or $\theta_{\max }$. For instance, with $k=3$, the probability to have a robot whose deformations are acceptable is greater than $88 \%$.

\subsection{Optimization Problem}

In order to speed up the optimization process, we imposed that the shapes of both proximal links are identical. Moreover, let us recall that, in order to have a good observation the distal links, these links must have a cylindrical shape and their diameter was fixed at $80 \mathrm{~mm}$. This is indeed not a problem, because these links are only solicited in tension/compression when efforts are applied in the plane of the robot motion and have very few deformation. So shape of their cross section has very few impact on the overall robot deformations.

Based on the previous developments and the specifications of Tab. 1, we formulated the following topology optimization problem:

$$
\begin{array}{cl}
\min _{\boldsymbol{\rho}} & z z_{1 R}=z z_{1}+m_{2} \ell_{1}^{2} \\
\text { under } & g_{1 i}=\left(\mathbf{u}_{d}^{2}\left(\mathbf{f}_{i}, \mathbf{q}^{*}, k\right)-u_{\max }^{2}\right) / u_{\max }^{2} \leq 0, \quad i=1,2 \\
& g_{2 i}=\left(\mathbf{u}_{d}^{2}\left(\mathbf{f}_{i}, \mathbf{q}^{*}, k\right)-\theta_{\max }^{2}\right) / \theta_{\max }^{2} \leq 0, \quad i=1,2
\end{array}
$$

where

- the objective function $z z_{1 R}=z z_{1}+m_{2} \ell_{1}^{2}$ is a grouping of inertial parameters $\left(z z_{1}\right.$ is the moment of inertia at point $A_{1}$ of the proximal link $\mathbf{u}_{e}^{\text {while }} m_{2}$ is the mass of the distal link). This term is usually preponderant in the dynamic model of the five-bar mechanism [39]. Thus minimizing it has a positive impact on the dynamic properties of the robot (decrease of the maximal torque, reduction of the energy consumed, etc).

- $\rho$ is the vector containing all decision variables $\rho_{i j}$.

- $\mathbf{q}^{*}$ are selected exciting configurations for the computation of the translational/rotational deformations when the loading $\mathbf{f}_{i}$ is applied $(i=1,2$ - these loadings are defined in Tab. 1). Configurations $\mathbf{q}^{*}$ can be found using an adequate methodology proposed in [33] and are the configurations where the deformations are the worst in the workspace. Thus, thanks to this approach, we can guarantee that the constraints are respected wherever in the workspace.

We solved this problem by using the optimization algorithm called the Linearization Method (LM) [32] which has proven, for our class of problems, to give results in a computational time equivalent to standard topology optimization algorithms [33]. 


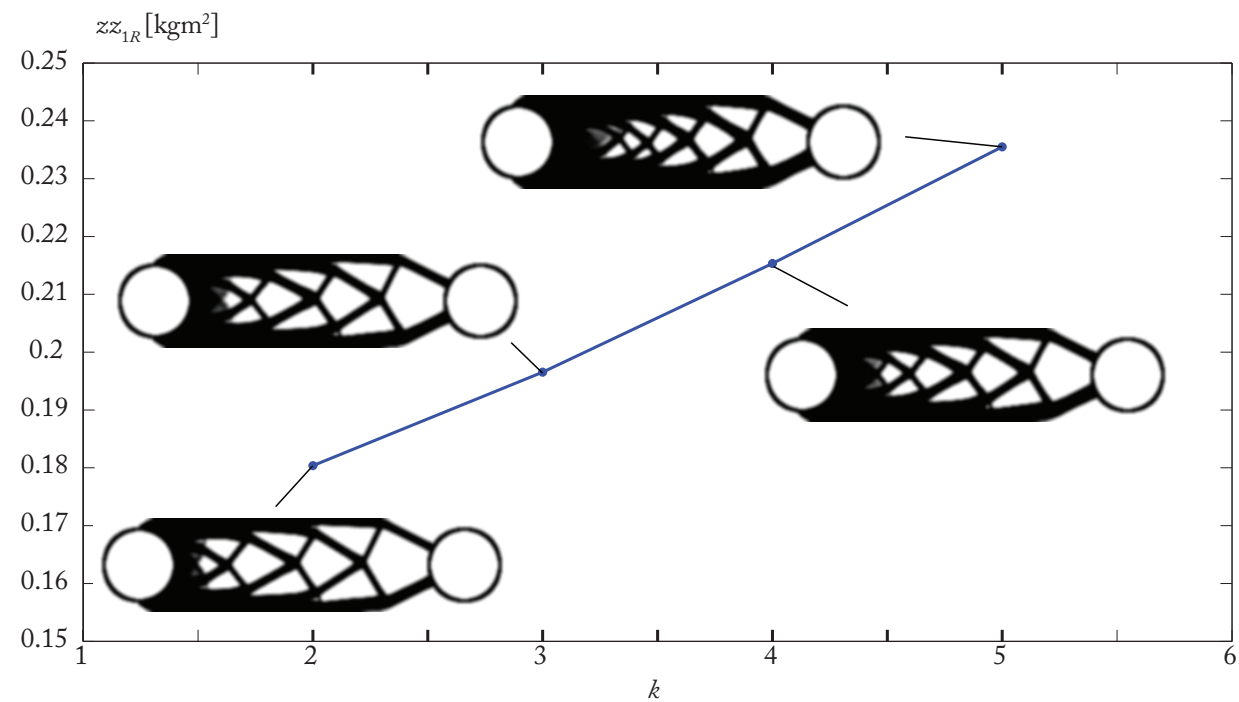

Figure 9: Design of the proximal links: final results for different values of $k$

\subsection{Result of the topology optimiza- tion}

The initial design domain for the proximal links is represented in Figure 8. Each link has two empty holes of diameters $6 \mathrm{~cm}$ at the extremity for the joints insertion (motor shaft at points $A_{i}$, and passive rotary joints for all others. For the meshing of the links, 22152 QUA4 planar elements of size $1 \times 1 \mathrm{~mm}$ are used and with $50 \mathrm{~mm}$ of thickness. All models and optimization algorithms have been encoded with Matlab in the Windows 7 environment. For a given value of $k$, computation time took around $5000 \mathrm{sec}$ (for a Pentium $42.70 \mathrm{GHz}, 16 \mathrm{~GB}$ of RAM).

The value of the objective function $z z_{1 R}$ as a function of $k$ is given in Fig. 9. The changes in the link design, when the value of $k$ increases, are essentially an increase of the material thickness near the hole at the "right-hand-side" of the link (connection with the distal link) and the presence of additional material near the "left-hand-side" of the link (connection with the actuator). The total inertia between tests with $k=2$ and $k=5$ is almost doubled, thus increasing the robustness of the link with respect to the wood variability, but also decreasing the robot dynamic performance.

We decided to finally achieve a prototype based on the results obtained for $k=3$. Key design features for this prototype are discussed in the next Section.

\section{CAD DESIGN AND PRO- TOTYPING}

Based on the previous optimization results, a prototype of a wooden five-bar mechanism was designed (Fig. 11). In this section, some key technological solution are highlighted:

Wooden distal links: are cylindrical for reason of facility of the observation with camera. They are partially hollowed out to limit their mass.

Wooden proximal links: are based on topology optimization results. They are build from 7 laser cut layers (7 mm thick), glued together with polyurethane glue. In order to improve the elastic behavior of these links along all directions, the layers are assembled by differently orienting the directions of the fibers for each layer, so that the links behave as they were made of a material with properties as close as possible from the isotropy. From top to bot- 


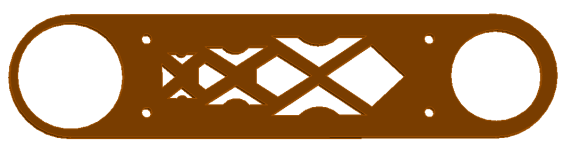

(a) Proximal Link: CAD Design

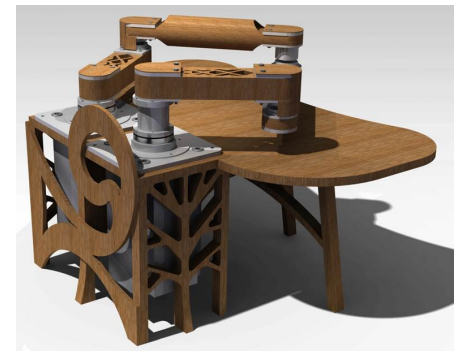

(b) Final Prototype

Figure 10: CAD Design of a wooden five-bar mechanism.

tom, angles between longitudinal axis of the link and wood grain ares $0^{\circ} ; 45^{\circ} ;-45^{\circ} ; 0^{\circ} ;-45^{\circ} ; 45^{\circ}, 0^{\circ}$. CAD design of the proximal links is shown in Fig. 10(a), it should be mentioned that the difference between the CAD model and the topology optimization results is due to local edge distances needed around the holes to ensure wood resistance, but the truss-like shape is kept.

Wooden chassis: is made with acetylated pine, and supports two direct drive actuators (SIMOTTICS S1FL6 servomotors) as shown in Fig. 10(b). Indeed, our robot should be able to fully rotate the proximal and distal links without interferences. Thus, we chose to set the actuators under the robot links.

Revolute joints: high precision angular contact ball bearings are used to design the revolute joints. This configuration delivers high level of stability and minimal backlash for the assembly.

FEM simulations of the CAD model were achieved in order to verify the overall deformation of the robot. Desired deformations under the loadings given in Tab. 1 were verified. We also tested the robot deformation under a loading of $10 \mathrm{~N}$ along $\mathbf{z}_{0}$ : results about 400 microns, which is acceptable.

At this step of the project RobEcolo, the wooden five-bar mechanism is built and future works will con-

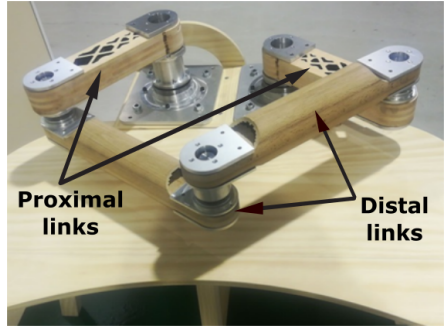

Figure 11: Final prototype of a wooden five-bar mechanism.

cern the experimental validations of the obtained theoretical results and the characterization of the robot properties.

\section{CONCLUSION}

In order to try to decrease the environmental impact of robot during their manufacturing phase, we investigated in this paper the possibility to design a wooden industrial robot whose performance in terms of accuracy and stiffness are guaranteed, even if exhibits significant dimensional ans mechanical variabilities. Thus, in order to be able to ensure stiffness and the accuracy of the wooden robot, two design methodologies are introduced in this paper. These two approaches are solved in cascade and aim at finding the robot optimal design architecture for which the impact of the wood properties variability is minimal. Based on the obtained architecture of robot, a prototype of a wooden five-bar mechanism is designed and built.

After the prototype validation phase, a first perspective of our work is to implement the sensor-based controllers. Then, several experiments for characterizing the robot stiffness and accuracy will be made to validate all the theoretical results obtained in the framework of the project RobEcolo.

\section{Acknowledgment}

This work was supported by the project RobEcolo funded by the French Région Pays de la Loire (Convention No. 2015-10773). 
The authors would like to thank Pauline Lafoux and Victoria Safyannikova for their work on the CAD design of the RobEcolo prototype.

\section{References}

[1] Fizians report: http://pagesperso.ls2n.fr/ briots/Project_Review.html.

[2] Thakur, V., 2013. Green Composites from Natural Resources. CRC Press.

[3] Laurent, T., Kergueme, J., Arnould, O., and Dureisseix, D., 2010. "Vers un robot en bois: Première partie". In French.

[4] Sharp, C., and Bowyer, M., 1995. Mosquito. Crecy Publishing.

[5] Fleming, P., Smith, S., and Ramage, M., 2014. "Measuring-up in timber: a critical perspective on mid-and high-rise timber building design". Architectural Research Quarterly.

[6] Roennau, A., Kerscher, T., and Dillmann, R., 2010. "Design and kinematics of a biologicallyinspired leg for a six-legged walking machine". In Proceedings of the 2010 3rd IEEE RAS \& EMBS ICBRB.

[7] Quigley, M., Asbeck, A., and Ng, A., 2011. "A low-cost compliant 7-DOF robotic manipulator". In Proceedings of the 2011 IEEE International Conference on Robotics and Automation (ICRA 2011).

[8] Kretschmann, D., 2010. Ch. 5: Mechanical properties of Wood. Forest Products Laboratory, United States Department of Agriculture Forest Service, Madison, Wisconsin.

[9] Wang, C., and Piao, C., 2011. "From hydrophilicity to hydrophobicity: a critical review".

[10] Chaumette, F., and Huchinson, S., 2008. Handbook of robotics. Springer, ch. 24: Visual Servoing and Visual Tracking, pp. 563-583.
[11] Kaci, L., Briot, S., Boudaud, C., and Martinet, P., 2016. "Control-based design of a five-bar mechanism". In Proceedings of the 6th European Conference on Mechanism Science (EuCoMeS 2016). Best Student Award Paper Finalist.

[12] Briot, S., Martinet, P., and Rosenzveig, V., 2015. "The hidden robot: an efficient concept contributing to the analysis of the controllability of parallel robots in advanced visual servoing techniques". IEEE Transactions on Robotics, 31(6), pp. 1337-1352.

[13] Angeles, J., and Park, F., 2008. Handbook of robotics. Springer, ch. Ch. 10: Performance Evaluation and Design Criteria.

[14] Asadpoure, A., Tootkaboni, M., and Guest, J., 2011. "Robust topology optimization of structures with uncertainties in stiffness - Application to truss structures". Computers and Structures, 89, pp. 1131-1141.

[15] Project RobEcolo: http://robecolo.irccyn.ecnantes.fr/.

[16] Campos, L., Bourbonnais, F., and Bonev, I., 2010. "Development of a five-bar parallel robot with large workspace". In Proceedings of the ASME 2010 International Design Engineering Technical Conferences and Computers and Information in Engineering Conference (IDETC/CIE 2010).

[17] Traslosheros, A., Sebastian, J., Angel, L., Roberti, F., and Carelliz, R., 2007. "Visual servoing of a parallel robot system". In IEEE ISISP, pp. 1-6.

[18] Andreff, N., Dallej, T., and Martinet, P., 2007. "Image-based visual servoing of gough-stewart parallel manipulators using legs observation". IJRR, 26(7), pp. 677-687.

[19] Andreff, N., and Martinet, P., 2006. "Visionbased kinematic modelling of some parallel manipulators for control purposes". In Proceedings of EuCoMeS. 
[20] Vignolo, A., Briot, S., Martinet, P., and Chen, C., 2014. "Comparative analysis of two types of leg-observation-based visual servoing approaches for the control of the five-bar mechanism". In Proceedings of the 2014 Australasian Conference on Robotics and Automation (ACRA 2014).

[21] Khalil, W., and Dombre, E., 2002. Modeling, Identification and Control of Robots. Hermes Penton London.

[22] Shi, B., Zhao, H., Ben-Ezra, M., Yeung, S., Fernandez-Cull, C., Shepard, R., Barsi, C., and Raskar, R., 2014. "Sub-pixel layout for superresolution with images in the octic group". In Proc. European Conference on Computer Vision $(\mathrm{ECCV})$.

[23] Merlet, J., 2006. Parallel Robots, 2nd ed. Springer.

[24] Briot, S., Glazunov, V., and Arakelian, V., 2013. "Investigation on the effort transmission in planar parallel manipulators". ASME JMR, 5(1).

[25] Sigmund, O., 2001. "A 99 line topology optimization code written in MATLAB". Structural and Multidisciplinary Optimization, 21(2), pp. $120-127$.

[26] Rossow, M., and Taylor, J., 1973. "A finite element method for the optimal design of variable thickness sheets". AIAA Journal, 11(11), pp. 1566-1569.

[27] Sigmund, O., and Maute, K., 2013. "Topology optimization approaches: A comparative review". Structural and Multidisciplinary Optimization, 48(6), pp. 1031-1055.

[28] Svanberg, K., 1987. "The method of moving asymptotes - a new method for structural optimization". Numerical Methods in Engineering, 24(2), pp. 359-373.

[29] Yin, L., and Yang, W., 2001. "Optimality criteria method for topology optimization under multiple constraints". Computers and Structures, 79(20-21), pp. 1839-1850.
[30] Chang, C., Borgart, A., Chen, A., and Hendriks, M., 2014. "Direct gradient projection method with transformation of variables technique for structural topology optimization". Structural and Multidisciplinary Optimization, $\quad \mathbf{4 9 ( 1 )}$, pp. 107-119.

[31] Fleury, C., 1989. "Conlin: an efficient dual optimizer based on convex approximation concepts". Structural Optimization, 1(2), pp. 81-89.

[32] Pshenichnyj, B., 1994. The Linearization Method for Constrained Optimization. Computational Mathematics. Springer.

[33] Briot, S., and Goldsztejn, A., 2018. "Topology optimization of industrial robots: Application to a five-bar mechanism". Mechanism and Machine Theory, 120, pp. 30-56.

[34] Bendsoe, M., and Sigmund, O., 1999. "Material interpolation schemes in topology optimization". Archive of Applied Mechanics, 69.

[35] Shabana, A., 2005. Dynamics of Multibody Systems. Cambridge University Press.

[36] Craig, R. R., and Bampton, M. C. C., 1968. "Coupling of substructures for dynamic analysis". AIAA Journal, $\boldsymbol{6}(7)$.

[37] Hill, C., 2006. Wood Modification: Chemical, Thermal and Other Processes. John Wiley \& Sons, Ltd.

[38] Kaci, L., Boudaud, B., Briot, S., and Martinet, P., 2017. "Elastostatic modelling of a wooden parallel robot". In Proceedings of the 7th IFToMM International Workshop on Computational Kinematics (CK2017).

[39] Pagis, G., Bouton, N., Briot, S., and Martinet, P., 2015. "Enlarging parallel robot workspace through type-2 singularity crossing". Control Engineering Practice, 39, pp. 1-11. 